INFLAMMATION

\section{NETs awaken}

sleeping cancer cells

Several studies have linked inflammation to the recurrence of cancer after periods of remission, but the reasons for this are not fully understood. Reporting in Science, Albrengues et al. show that inflammation-induced release of neutrophil extracellular traps (NETs) can promote the awakening of dormant cancer cells by remodelling the extracellular matrix (ECM).

To explore how inflammation affects dormant cancer cells, the authors injected labelled breast cancer cells from the mouse D2.0R or human MCF7 cell lines into syngeneic $\mathrm{BALB} / \mathrm{c}$ mice or nude mice, respectively. Injection of these cells did not lead to the formation of tumours (even after 240 days) but single, non-proliferative cancer cells were detected in the lungs. A single intranasal application of lipopolysaccharide (LPS) did not awaken the dormant D2.0R or MCF7 cells; however, three applications of LPS at 3-day intervals led to aggressive lung metastasis.

LPS treatment was associated with marked neutrophil infiltration and re-entry of dormant cancer cells into the cell cycle. Notably, neutrophil depletion prevented the LPS-induced awakening of dormant cancer cells. Neutrophil activation is associated with the production of neutrophil extracellular traps (NETs), which are scaffolds of DNA containing cytotoxic proteins and proteases. As many NETs were detected in the lungs following LPS treatment, the authors tested whether these promote the awakening of dormant cancer cells. Indeed, blockade of NETs (using a protein-arginine deiminase type 4 (PAD4) inhibitor or through DNase I-mediated digestion) inhibited awakening of dormant D2.0R or MCF7 cells in response to LPS treatment. LPS-induced NET formation was also linked to lung metastasis in models of prostate and breast cancer. Furthermore, the authors showed that exposure of mice to tobacco smoke could also awaken dormant cancer cells via NET formation.

These findings indicated that inflammation-induced NET formation awakens dormant cancer cells in a number of different mouse models; the authors next used 3D culture systems to examine the mechanistic basis of this. They found that NETs promote cancer cell awakening in vitro in the presence of an artificial ECM, but this could be blocked by inhibiting neutrophil elastase (NE) and matrix metalloproteinase 9 (MMP9), which are NET-associated proteases. Inhibition of NE and MMP9 also prevented LPS-induced cancer recurrence in vivo and further experiments in vitro suggested that these NET-associated proteases promote awakening of dormant cancer cells by remodelling the ECM.

Both NE and MMP9 were necessary for awakening cancer cells and the authors showed this was due to their sequential cleavage of laminin-111, an ECM component that has previously been linked with the regulation of cell proliferation. The authors showed that laminin-111 is present in mouse lung tissue and cleaved following intranasal LPS treatment; in vitro experiments suggested that NET-associated proteases also cleave other laminin isoforms to support the awakening of dormant cancer cells. Meanwhile, DNA released from NETs was shown to act as a scaffold to promote the cleavage of laminin by NET-associated proteases.

The authors showed that NET remodelling of ECM promotes the awakening of dormant cancer cells by driving $\alpha 3 \beta 1$ integrin signalling in cancer cells. They hypothesized that proteolytic remodelling of the ECM leads to the emergence of a new epitope in laminin that is sensed by $\alpha 3 \beta 1$ integrin to promote cancer cell proliferation. In support of this idea, the authors generated an antibody (Ab28) that recognizes an epitope present in NET-remodelled, but not intact, laminin and showed that this epitope only emerges in the lungs after exposure to LPS or tobacco smoke and co-localizes with proliferating cancer cells. Furthermore, treatment of mice with Ab28 prevented the awakening of dormant cancer cells following LPS treatment or tobacco smoke exposure.

In summary, this study shows how tissue inflammation can support the recurrence of cancer after periods of dormancy. Moreover, it suggests that NETs could be targeted to prevent the re-emergence of cancer in patients who are in remission.

Yvonne Bordon

ORIGINAL ARTICLE Albrengues, J. et al. Neutrophil extracellular traps produced during inflammation awaken dormant cancer cells in mice. Science 361, eaao4227 (2018) 